\title{
Comprendre l'intérêt public de la Colombie- Britannique lié aux opioïdes durant la crise: une exploration axée sur Google Trends sur le comportement en matière de recherche de l'information en ligne concernant la santé
}

\author{
Par C. Patterson, BSc, MD, and M.J. Douma, RN, MN, ENC(C), CCNC(C), CCN(C)
}

\section{Introduction}

A u cours des deux dernières décennies, la morbidité et la mortalité liées aux opioïdes ont dégénéré pour devenir une urgence en santé publique (Ayers, Ribisl et Browstien, 2011). Le Canada a la deuxième plus forte consommation d'opioïdes par habitant au monde (BC Drug Overdose \& Alert Partnership, 2014). Au Canada, les opioïdes sans ordonnance sont la deuxième substance la plus souvent utilisée par les adolescents et les adultes, après le cannabis (Burritt, 2015). En 2016, les taux de mortalité des opioïdes en Alberta et en Colombie-Britannique étaient supérieurs à 14 pour 100000 habitants, soit plus que les homicides, les suicides, les cancers du sein et du côlon (Busse, Craigie, Juurlink, Buckley, Wang, Couban, et al. 2017). Les lignes directrices canadiennes sur l'utilisation des opiö̈des ont été mises à jour en 2017 pour tenir compte des préoccupations relatives à l'utilisation des opiö̈des (Association des pharmaciens et pharmaciennes du Canada, 2017). En réponse à l'augmentation des surdoses, les gouvernements provinciaux ont pris l'habitude d'augmenter l'accès à la naloxone. L’Alberta, l'Ontario, la Nouvelle-Écosse, les Territoires $\mathrm{du}$ Nord-Ouest et le Yukon ont rendu la naloxone gratuite et accessible à tous dans les pharmacies ou les centres de distribution communautaires (CCENDU, 2016, Fischer, Kurdyak, Goldner, Tyndall et Rehm, 2016). Les professionnels de la santé, tels que le personnel des services d'urgence, sont à la recherche d'informations pour faire face à l'escalade de la crise.

\section{Présentation de Google Trends}

En plus de fournir de l'information sur la morbidité et la mortalité liées aux opioïdes et sur l'accès aux programmes de naloxone à emporter au Canada, le moteur de recherche Internet Google fournit des données pertinentes dans l'analyse du personnel des urgences. Google Trends a déjà été utilisé pour surveiller le comportement du public en matière de santé (Fischer, Murphy, Rudzinski et MacPherson, 2016). De plus, des données de recherche Google ont été utilisées pour décrire le comportement actuel et futur (Goel, Hofman, Lahaie, Pennock et Watts, 2010). Les exemples comprennent le suivi des éclosions de maladies transmissibles et, plus récemment, l'examen de la popularité des systèmes électroniques d'administration de nicotine et de l'utilisation illicite de méphédrone aux États-Unis (Santé Canada, 2018, International Narcotics Control Board, 2017).
Le but de cet document est de décrire comment Google Trends peut être utilisé par le personnel des urgences pour explorer d'importants phénomènes liés à la santé. Notre exploration comprend : i) une analyse de la recherche sur Internet et des nouvelles concernant le fentanyl et la naloxone, ainsi que ii) une analyse corrélationnelle de base des demandes de fentanyl et de la naloxone avec les données sur la mortalité en ColombieBritannique. Nous espérons démontrer comment les données ouvertes peuvent augmenter les initiatives de surveillance, décrire l'intérêt du public vis-à-vis des sujets liés à la santé et leur comportement en matière de recherche des connaissances.

\section{Méthodes}

\section{Sources de données}

Les données ont été obtenues à partir de Google Trends (www. google.ca/trends), une base de données en temps réel et gratuite. Les données de recherche sur le Web et les nouvelles de Google Trends liées au fentanyl et au naloxone ont été consultées le 1er septembre 2017. Les données ont été extraites de janvier 2012 à la fin de juillet 2017 et se limitaient au volume de recherche de la Colombie-Britannique. Les données sur la mortalité de 2012 à 2017 proviennent du rapport du Bureau du coroner de la Colombie-Britannique (COBC) sur le nombre de décès où le fentanyl a été détecté, ou ii) comme cause unique de décès entre 2014 et 2017 (http://www2.gov.bc.ca/gov/content/safety/ public-safety/death-investigation/statistical-reports).

\section{Stratégie de recherche et format des données}

Les données de recherche provenant de Google Trends sont fournies sous la forme d'un volume de recherche relative (VRR) qui est mis à l'échelle à la période du volume de recherche le plus élevé selon la période de temps demandée (VRR 100). Tous les autres volumes de recherche sont indiqués en tant que volume relatif du maximum signalé (par exemple, $50 \%$ de la proportion de recherche maximale apparaîtraient comme 50). La catégorie de données « santé » a été sélectionnée et les recherches liées au fentanyl et à la naloxone ont été comparées simultanément sur la même échelle VRR. Le nom générique de chaque médicament a été utilisé comme terme de recherche en combinaison avec les modificateurs de termes de recherche proposés par Google. Les termes de recherche associés ont été générés automatiquement par Google Trends et inclus dans cette analyse pour les recherches sur le Web et les actualités. 


\section{Analyses statistiques}

Les données Google Trends ont été téléchargées pour une analyse statistique de base (y compris les corrélations de Pearson) en utilisant Microsoft Excel (Redmond, Washington, ÉtatsUnis). Une exemption de l'évaluation éthique institutionnelle a été obtenue en raison de la nature à source ouverte des données utilisées pour l'analyse.

\section{Résultats}

Résultats des recherches web sur le Fentanyl

De janvier 2012 à août 2014, le fentanyl a été rarement consulté, avec un VRR inférieur à 10. Les recherches sur le fentanyl ont atteint un VRR de 27 en mars 2015. Le volume de recherche sur le fentanyl a atteint un sommet en août 2015, indiquant une plus forte proportion de recherche sur le fentanyl, ou la naloxone, de janvier 2012 à juillet 2017. Le VRR sur le fentanyl est demeuré élevé mais stable (fluctuant entre 15 et 37 VRR) de septembre 2015 à août 2016, puis a atteint un VRR de 81 en septembre 2016. Les villes de Colombie-Britannique ayant les plus gros volumes de fentanyl, de janvier 2012 à la fin de juillet 2017, étaient Maple Ridge (VRR de 100), suivie de Delta (VRR de 84), de Kamloops (VRR de 81) et de Kelowna (VRR de 81). Voir la figure 1 pour un graphique du fentanyl par rapport au volume de recherche sur la naloxone.

\section{Résultats des recherches de nouvelles sur le Fentanyl}

Les recherches de nouvelles liées au fentanyl sont restées faibles (VRR moyen de quatre) de janvier 2012 à juillet 2015, avec un seul pic VRR de 13 entre juillet et décembre 2013. En août 2015, il y a eu un pic de recherche de nouvelles liées au fentanyl (VRR de 100). Depuis lors, les volumes de recherche liés au fentanyl sont demeurés constamment élevés (VRR de 11 à 72 , moyenne de 32 , soit une augmentation de $889 \%$ ) de septembre 2015 à juillet 2017. Voir la figure 2 pour un graphique sur le fentanyl contre le volume de recherche de nouvelles sur la naloxone.

\section{Résultats des recherches web sur la naloxone}

Les résultats de la recherche pour la naloxone étaient faibles de février 2012 à janvier 2016. De janvier 2016 à juillet 2017, le VRR moyen pour les recherches sur la naloxone était de neuf et le VRR n'était pas inférieur à quatre.

Le volume de recherche pour la naloxone est demeuré élevé de janvier 2016 à juillet 2017. Kelowna (VRR de 100), Vancouver (VRR de 65), Surrey (VRR de 55) et Victoria (VRR de 54)).

Volume de recherche relative sur le Web pour le fentanyl, l'oxycodone et la naloxone en Colombie-Britannique entre le $1^{\text {er }}$ janvier 2012 et le $1^{\text {er }}$ juillet 2017

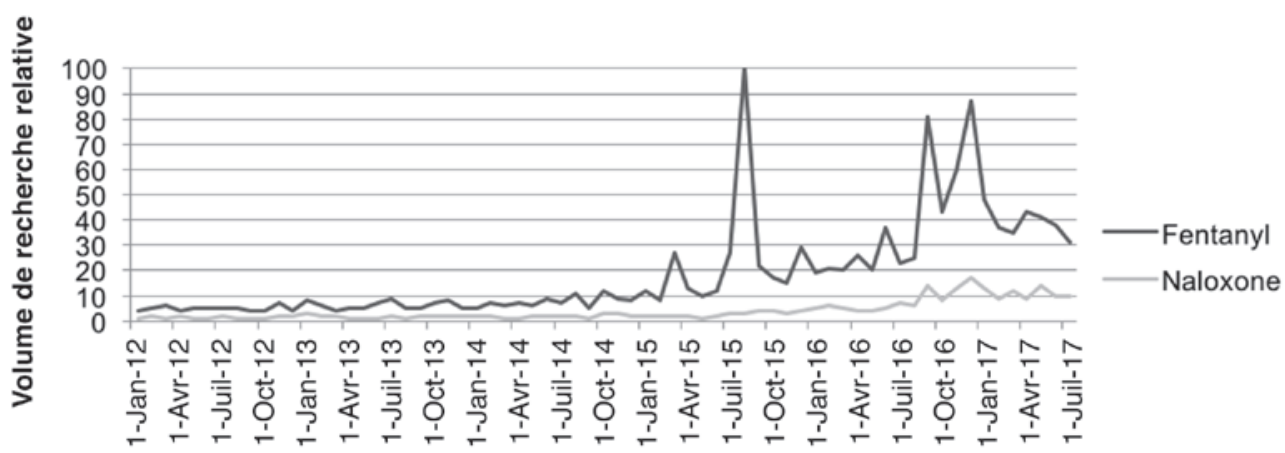

Figure 1: Volume de recherche relative sur le Web pour le fentanyl, l'oxycodone et la naloxone entre le $1^{\text {er }}$ janvier 2012 et le $1^{\text {er }}$ juillet 2017. Source de données: Google Trends (www.google.com/ trends)

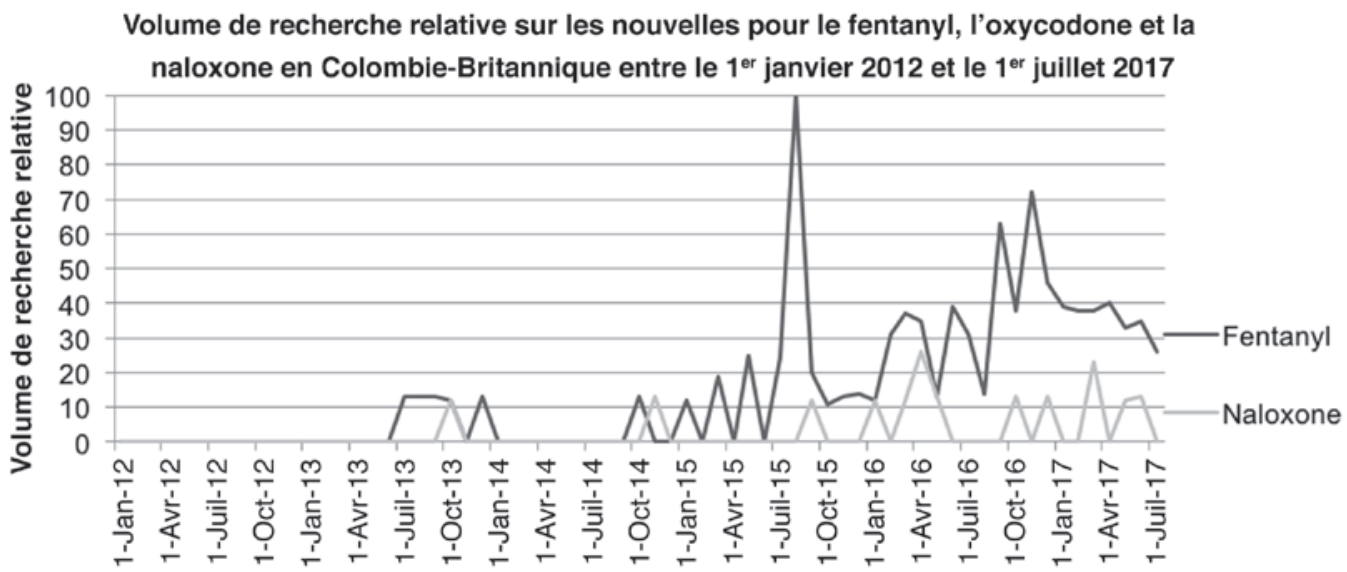

Figure 2: Volume de recherche relative sur les nouvelles pour le fentanyl, l'oxycodone et la naloxone entre entre le $1^{\text {er }}$ janvier 2012 et le $1^{\text {er }}$ juillet 2017. Source de données: Google Trends (www.google.com/trends) 


\section{Résultats des recherches de nouvelles sur la naloxone}

Les recherches de nouvelles sur la naloxone sont demeurées systématiquement faibles (VRR de trois) au cours de la période de recherche. Il n'a pas été possible d'analyser les données de recherche de nouvelles pour la naloxone par lieu en raison de faibles volumes de recherche.

\section{Termes de recherche connexes}

La sortie de données Google Trends inclut une liste de termes de recherche connexes qui sont recherchés conjointement avec le mot clé choisi. Les termes de recherche connexes pour le fentanyl et la naloxone comprenaient des variations orthographiques, des questions sur les effets, les effets secondaires et la dépendance. Les recherches les plus fréquentes sur le fentanyl comprenaient « timbre fentanyl » (VRR de 10), « surdose de fentanyl $\gg($ VRR de 10), « Vancouver fentanyl $\gg($ VRR de 10), « qu'est-ce que le fentanyl » (VRR de cinq) et « effets du fentanyl $\gg($ VRR de cinq).

\section{Fentanyl, naloxone et exploration des données BEP}

Le nombre de décès dans lesquels le fentanyl était soit la seule cause, soit détecté lors du dépistage toxicologique en association avec d'autres médicaments, a augmenté régulièrement depuis 2008. Cependant, le manque de données normalisées fiables à travers le pays rend la mortalité du fentanyl difficile à commenter. Les données de 2012 à 2016 sont disponibles en Saskatchewan, Alberta et en Colombie-Britannique. Cependant, seule la Colombie-Britannique a déclaré des taux de mortalité mensuels (voir le tableau 1 pour les données).

Les volumes de recherche sur le Web et les nouvelles sur Google pour le fentanyl étaient fortement corrélés (Pearson 0,92). Les volumes de recherche sur le Web liés à la naloxone et les données de mortalité $\mathrm{BEP}$ sont également fortement corrélatives

\section{Tableau 1 : Mortalité mensuelle liée au fentanyl en} Colombie-Britannique de janvier 2012 à juillet 2017

\begin{tabular}{|l|c|c|c|c|c|c|}
\hline Date & $\mathbf{2 0 1 2}$ & $\mathbf{2 0 1 3}$ & $\mathbf{2 0 1 4}$ & $\mathbf{2 0 1 5}$ & $\mathbf{2 0 1 6}$ & $\mathbf{2 0 1 7}$ \\
\hline Jan. & 0 & 5 & 5 & 20 & 46 & 102 \\
\hline Févr. & 0 & 3 & 5 & 8 & 29 & 101 \\
\hline Mars & 0 & 6 & 9 & 8 & 48 & 112 \\
\hline Avr. & 1 & 8 & 8 & 12 & 48 & 117 \\
\hline Mai & 1 & 3 & 8 & 8 & 37 & 106 \\
\hline Juin & 1 & 2 & 6 & 11 & 43 & 95 \\
\hline Juill. & 0 & 1 & 3 & 14 & 40 & 73 \\
\hline Août & 1 & 4 & 8 & 15 & 37 & \\
\hline Sept. & 1 & 2 & 9 & 15 & 41 & \\
\hline Oct. & 0 & 4 & 13 & 16 & 53 & \\
\hline Nov. & 4 & 6 & 6 & 12 & 106 & \\
\hline Déc. & 3 & 6 & 11 & 12 & 129 & \\
\hline Total & $\mathbf{1 2}$ & $\mathbf{5 0}$ & $\mathbf{9 1}$ & $\mathbf{1 5 1}$ & $\mathbf{6 5 7}$ & $\mathbf{7 0 6}$ \\
\hline
\end{tabular}

(Pearson 0.92). Cependant, les volumes de recherche sur le Web et les nouvelles sur Google pour le fentanyl et la naloxone nétaient que modérément linéairement corrélés aux données de mortalité BEP, de janvier 2012 à juillet 2017 (Pearson 0,7 et 0,67 , respectivement). Il y avait une corrélation minimale entre les volumes de recherche sur le Web et les nouvelles pour la naloxone (Pearson 0,40).

\section{Discussion}

Résultats des recherches sur le web et les nouvelles

Les recherches sur Internet pour le fentanyl n'ont pas augmenté significativement jusqu'en août 2015. Cette information, avec la forte corrélation (Pearson 0.96) entre les VRR du fentanyl dans les recherches sur le Web et les nouvelles, et la corrélation plus faible entre la mortalité associée au fentanyl et les recherches sur le Web $(0,70)$, peut suggérer que les recherches sur Internet pour le fentanyl peuvent être davantage motivées par les informations des médias que par les taux de mortalité. Laugmentation des recherches sur le Web pour la naloxone entre août 2015 et juillet 2017 n'a pas été associée à une augmentation des recherches de nouvelles. La forte corrélation entre la recherche de naloxone sur le Web et l'augmentation de la mortalité (Pearson 0,92) au cours de la période étudiée et l'intérêt manifeste pour la naloxone en dépit de l'absence d'attention médiatique peuvent représenter une augmentation du comportement en matière de recherche de l'information et non une réponse aux nouvelles et médias. Cependant, c'est une conjecture de notre part et nécessite une validation.

La hausse du volume de recherche liée au fentanyl en août 2015 coïncide avec des décès tragiques et très médiatisés. Il s'agit notamment de Jack Bodie, 17 ans (King, Fraser, Boikos, Richardson et Harper, 2014) et de jeunes parents, Hardy et Amelia Leighton, qui ont rendu orphelin leur enfant de deux ans après une intoxication au fentanyl (Sienuid \& Woo, 2015). Il a déjà été démontré que les décès médiatisés influencent les nouvelles liées à la santé et les recherches sur Internet (Waszak et Kawalec, 2017). Ces événements exigent de la sensibilité, mais représentent également une opportunité importante pour le calendrier des campagnes de promotion de la santé et la collecte de fonds, surtout lorsque l'intérêt du public est le plus élevé.

\section{Termes de recherche connexes}

Les Centers for Disease Control de la Colombie-Britannique signalent qu'avant 2014 les timbres de fentanyl étaient la source la plus courante de fentanyl mal utilisé et que l'extraction du contenu du timbre pour l'injection intraveineuse était la voie d'administration la plus courante (16). Ceci peut expliquer que le « timbre de fentanyl » soit l> un des termes de recherche connexes les plus couramment utilisés. Des termes de recherche supplémentaire, comme « surdosage de fentanyl », « fentanyl marijuana attachée » et « carfentanil », représentent des sujets $\mathrm{d}$ >intérêt pour les professionnels de la santé à aborder dans leur enseignement et leur programmation. Les termes de recherche connexes à la naloxone (y compris la « dose de narcane » et le «trousse de narcane») suggèrent que les chercheurs sont probablement à la recherche d'informations sur les manières d'inverser le surdosage d'opioïdes. 


\section{Fentanyl, naloxone et explorations des données BEP}

La forte corrélation linéaire entre les volumes de recherche sur le Web et les nouvelles sur Google pour le fentanyl pourrait représenter la gravité et la médiatisation de la crise du fentanyl. La relation toute aussi forte entre les volumes de recherche sur le web de la naloxone et les données de mortalité liées au fentanyl BEP est une conclusion notable de notre recherche exploratoire. Logiquement, on peut conclure que, comme plus de décès ont eu lieu, l'intérêt pour la prévention de décès supplémentaires a augmenté, mais les essais statistiques effectués ne peuvent pas ajouter de poids à cette allégation. C'est une hypothèse intéressante qui nécessite d'autres essais.

\section{Limites}

Ce document présente des limites importantes, car toutes les données de Google Trends sont générées en proportion au volume total de recherche, ce qui signifie qu'il existe un manque de données concrètes sur le nombre de recherches effectuées, ce qui limite les comparaisons possibles. De plus, le manque de données normalisées sur la mortalité liée au fentanyl dans les provinces limite considérablement les conclusions qui peuvent en être tirées. Enfin, comme les recherches sur le Web génèrent encore un certain contenu médiatique, il est difficile de faire la différence entre un comportement en matière de de recherche de l'information et un comportement en matière de recherche de santé uniquement à travers Google Trends. Notre recherche exploratoire se limite à être descriptive et génératrice d'hypothèses.

\section{Conclusions}

En conclusion, bien que la portée de cette étude ait été limitée, elle a démontré comment Google Trends peut être utilisé pour accéder à des données de volume de recherche à jour. Les intérêts vis-à-vis du fentanyl et de la naloxone ont été démontrés. Enfin, il est possible d'améliorer l'utilité des requêtes sur le Web de Google en matière de santé en incluant des informations sur les premiers secours en cas de surdosage de fentanyl avec des recherches sur le fentanyl ou la naloxone.

\section{Au sujet des auteurs}

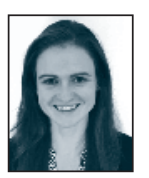

Christine Patterson est étudiante en troisième année en médecine à l'Université de l'Alberta et s'intéresse aux toxicomanies et à la médecine d'urgence. Elle a complété un baccalauréat en pharmacologie à l'Université de l'Alberta en 2014 et a déjà travaillé dans le domaine de la réadaptation. Elle se concentre actuellement sur l'achèvement de sa troisième année d'école de médecine en tant qu'externat communautaire intégré à Whitecourt, en Alberta.

\section{RÉFÉRENCES}

Ayers, J.W., Ribisl, K M., \& Brownstein, J.S. (2011). Tracking the rise in popularity of electronic nicotine delivery systems (electronic cigarettes) using search query surveillance. American Journal of Preventive Medicine, 40(4), 448-453.

British Columbia Drug Overdose \& Alert Partnership Report. (2014). BCDrug Use Epidemiology. Retrieved from: http://www.bccdc.ca/ resource-gallery/Documents/Statistics\%20and\%20Research/ Publications/Epid/Other/FinalDOAPReport2014.pdf

Burritt, D. (2015). Hardy and Amelia Leighton's death due to fentanyl, other drugs, coroner says. CBC News. Retrieved from http:// www.cbc.ca/news/canada/british-columbia/hardy-andamelia-leighton-s-death-due-to-fentanyl-other-drugs-coronersays- 1.3173286

Busse, J.W., Craigie, S., Juurlink, D.N., Buckley, D.N., Wang, L., Couban, R.J., ... Cull, C. (2017). Guideline for opioid therapy and chronic non-cancer pain. Canadian Medical Association Journal, 189(18), E659-E666.

Canadian Pharmacists Association. (August 2017). Environmental scan: Access to naloxone across Canada. Retrieved from: https:// www.pharmacists.ca/cpha-ca/assets/File/cpha-on-theissues/Environmental\%20Scan $\% 20-\% 20$ Access $\% 20$ to $\% 20$ Naloxone\%20Across\%20Canada_Final.pdf

Carneiro, H.A., \& Mylonakis, E. (2009). Google Trends: A webbased tool for real-time surveillance of disease outbreaks. Clinical Infectious Diseases, 49(10), 1557-1564.

CCENDU. (2016, March). The availability of take-home naloxone in Canada. Canadian Centre on Substance Abuse. Retrieved from: http://www.ccsa.ca/Resource\%20Library/CCSA-CCENDUTake-Home-Naloxone-Canada-2016-en.pdf

Fischer, B., Kurdyak, P., Goldner, E., Tyndall, M., \& Rehm, J. (2016). Treatment of prescription opioid disorders in Canada: Looking at the 'other epidemic'? Substance Aabuse Treatment, Prevention, and
Policy, 11(1), 12.

Fischer, B., Murphy, Y., Rudzinski, K., \& MacPherson, D. (2016). Illicit drug use and harms, and related interventions and policy in Canada: A narrative review of select key indicators and developments since 2000. International Journal of Drug Policy, 27, 23-35.

Goel, S., Hofman, J.M., Lahaie, S., Pennock, D.M., \& Watts, D.J. (2010). Predicting consumer behavior with Web search. Proceedings of the National Academy of Sciences, 107(41), 17486-17490.

Health Canada. (2018, January 15). National report: Apparent opioidrelated deaths (2016). Retrieved from https://www.canada.ca/ en/health-canada/services/substance-abuse/prescription-drugabuse/opioids/national-report-apparent-opioid-related-deaths. html

International Narcotics Control Board. (2017). Estimated world requirements of narcotic drugs for 2017. Narcotic Drugs Narcotic Drugs 2016, 267-308. doi:10.18356/9c258508-en-fr-es

King, N.B., Fraser, V., Boikos, C., Richardson, R., \& Harper, S. (2014). Determinants of increased opioid-related mortality in the United States and Canada, 1990-2013: A systematic review. American Journal of Public Health, 104(8). doi:10.2105/ajph.2014.301966

Sienuid K., \& Woo, A. (2015, August 15). B.C overdose highlights small number of recreational fentanyl users who seek out the powerful drug. The Globe and Mail. Retrieved from https://www.theglobeandmail.com/news/british-columbia/ bc-seeing-rise-in-drug-users-unknowingly-ingesting-fentanyl/ article25842305/

Waszak, P.M., \& Kawalec, N. (2017). Impact of celebrities' cancer deaths on oncology-related news and internet searches in Poland. Public health, 144, 13-15.

Yin, S., \& Ho, M. (2012). Monitoring a toxicological outbreak using Internet search query data. Clinical toxicology, 50(9), 818-822. 